\title{
Near-infrared detection and characterization of the exoplanet HD 95086 b with the Gemini Planet Imager ${ }^{\star}$
}

\author{
R. Galicher ${ }^{1}$, J. Rameau ${ }^{2}$, M. Bonnefoy ${ }^{2}$, J.-L. Baudino ${ }^{1}$, T. Currie ${ }^{3}$, A. Boccaletti ${ }^{1}$, G. Chauvin ${ }^{2}$, \\ A.-M. Lagrange ${ }^{2}$, and C. Marois ${ }^{4}$ \\ 1 LESIA, CNRS, Observatoire de Paris, Univ. Paris Diderot, UPMC, 5 place Jules Janssen, 92190 Meudon, France \\ e-mail: raphael.galicher@obspm.fr \\ 2 UJF-Grenoble 1/CNRS-INSU, Institut de Planétologie et d'Astrophysique de Grenoble (IPAG) UMR 5274, 38041 Grenoble, France \\ 3 Department of Astronomy and Astrophysics, Univ. of Toronto, 50 St. George St., Toronto M5S 1A1, Canada \\ ${ }^{4}$ National Research Council of Canada Herzberg, 5071 West Saanich Road, Victoria V9E 2E7, Canada
}

Received 18 March 2014 / Accepted 15 April 2014

\begin{abstract}
HD 95086 is an intermediate-mass debris-disk-bearing star. VLT/NaCo $3.8 \mu$ m observations revealed it hosts a $5 \pm 2 M_{\text {Jup }}$ companion (HD $95086 \mathrm{~b}$ ) at $\simeq 56 \mathrm{AU}$. Follow-up observations at 1.66 and $2.18 \mu \mathrm{m}$ yielded a null detection, suggesting extremely red colors for the planet and the need for deeper direct-imaging data. In this Letter, we report $H$ - $(1.7 \mu \mathrm{m})$ and $K_{1}-(2.05 \mu \mathrm{m})$ band detections of HD 95086 b from Gemini Planet Imager (GPI) commissioning observations taken by the GPI team. The planet position in both spectral channels is consistent with the NaCo measurements and we confirm it to be comoving. Our photometry yields colors of $H-L^{\prime}=3.6 \pm 1.0 \mathrm{mag}$ and $K_{1}-L^{\prime}=2.4 \pm 0.7 \mathrm{mag}$, consistent with previously reported 5- $\sigma$ upper limits in $H$ and $K$ s. The photometry of HD $95086 \mathrm{~b}$ best matches that of 2M $1207 \mathrm{~b}$ and HR $8799 \mathrm{cde}$. Comparing its spectral energy distribution with the BT-SETTL and LESIA planet atmospheric models yields $T_{\text {eff }} \sim 600-1500 \mathrm{~K}$ and $\log g \sim 2.1-4.5$. Hot-start evolutionary models yield $M=5 \pm 2 M$ Jup Warm-start models reproduce the combined absolute fluxes of the object for $M=4-14 M_{\text {Jup }}$ for a wide range of plausible initial conditions ( $S_{\text {init }}=8-13 k_{\mathrm{B}} /$ baryon). The color-magnitude diagram location of HD 95086 b and its estimated $T_{\text {eff }}$ and $\log g$ suggest that the planet is a peculiar $L-T$ transition object with an enhanced amount of photospheric dust.
\end{abstract}

Key words. planets and satellites: atmospheres - planets and satellites: detection - stars: individual: HD 95086 -

instrumentation: adaptive optics

\section{Introduction}

HD $95086 \mathrm{~b}$ is a directly imaged planet $\left(5 \pm 2 M_{\mathrm{J}}, a_{\text {proj }}=55.7 \pm\right.$ 2.5 AU) discovered by Rameau et al. (2013a) in $L^{\prime}(3.8 \mu \mathrm{m})$ with VLT/NaCo (Lenzen et al. 2003; Rousset et al. 2003) orbiting the young A8 star HD $95086\left(M \sim 1.6 M_{\odot}\right)$, a member of the Lower Centaurus Crux subgroup (17 \pm 4 Myr, Pecaut et al. 2012; Meshkat et al. 2013). Additional $L^{\prime}$ images taken later in 2013 confirmed that the object is comoving with its star (Rameau et al. 2013b).

$\mathrm{NaCo} K \mathrm{~s}(2.18 \mu \mathrm{m})$ and NICI (Chun et al. 2008) $H$-band $(1.65 \mu \mathrm{m})$ observations failed to reveal the planet (Rameau et al. 2013a; Meshkat et al. 2013). However, $5 \sigma$ lower limits of $\mathrm{Ks}-\mathrm{L}^{\prime}=1.2 \pm 0.5 \mathrm{mag}$ and $H-L^{\prime}=3.1 \pm 0.5$ mag suggest that the planet may have extremely red colors, similar to the young planets HR 8799 bcde and 2M 1207 b (Chauvin et al. 2004; Marois et al. 2008, 2010a), which have very dusty/cloudy atmospheres (Barman et al. 2011; Currie et al. 2011). Higher contrast near-IR data able to detect HD $95086 \mathrm{~b}$ can provide better comparisons with these objects and better constrain its atmosphere.

In this Letter, we present detections of HD 95086b with the recently installed Gemini Planet Imager (GPI, Macintosh et al. 2014) on Gemini South from public data as a part of GPI commissioning observations (Perrin et al. 2014). The data (acquired

* Based on public data taken at the GPI commissioning. and reduced by the GPI team), their analysis, and the detections are presented in Sect. 2. In Sect. 3, we combine GPI $H$ and $K_{1}$ photometry with $\mathrm{NaCo} L^{\prime}$ photometry to constrain the physical properties of HD $95086 \mathrm{~b}$.

\section{Observations and data reduction}

The GPI is a new instrument for imaging and characterizing planets around young nearby bright stars, combining an extreme adaptive optics system, coronagraphs, and an integrated field spectrograph (IFS). The IFS platescale is $14.3 \pm 0.3$ mas px $^{-1}$ for a $2.8^{\prime \prime}$ field-of-view (FOV) and the true North position angle is given within $1 \mathrm{deg}^{1}$.

The HD 95086 spectral data were obtained at $\mathrm{H}(1.5-1.8 \mu \mathrm{m}$, $R=44-49)$ and $K_{1}(1.9-2.19 \mu \mathrm{m}, R=62-70)$ in 2013 December using apodized Lyot coronagraphs (Table 1) and angular differential imaging (ADI, Marois et al. 2006a). Conditions were good: $0.43^{\prime \prime}$ and 0.6" DIMM seeing, air masses of 1.32 and 1.34, and coherence times of $19 \mathrm{~ms}$ and $17 \mathrm{~ms}$, respectively. The GPI commissioning team used their pipeline for badpixel removal, destriping, non-linearity and persistence corrections, flat-fielding, wavelength calibration, and converting the data into spectral data cubes. We used the data cubes relying on the GPI pipeline quality. The data are made of 21 and 17 spectral

1 http://planetimager.org/ 
Table 1. Observing log of HD 95086 with GPI.

\begin{tabular}{lccccc}
\hline \hline Date & Filter & Coro mask diam $($ mas) & DIT(s) $\times$ NDITS $\times \mathrm{Nb}_{\lambda}$ & Nb images & FOV rotation $\left(^{\circ}\right)$ \\
\hline $2013 / 12 / 10$ & $K_{1}$-coro & 306 & $119.29278 \times 1 \times 37$ & 17 & 11.7 \\
$2013 / 12 / 11$ & $H$-coro & 246 & $119.29278 \times 1 \times 37$ & 21 & 15.0 \\
\hline
\end{tabular}

Notes. Date, filter, occulting mask diameter, exposure, numbers of coadds, of spectral channels, of images, and FOV rotation.
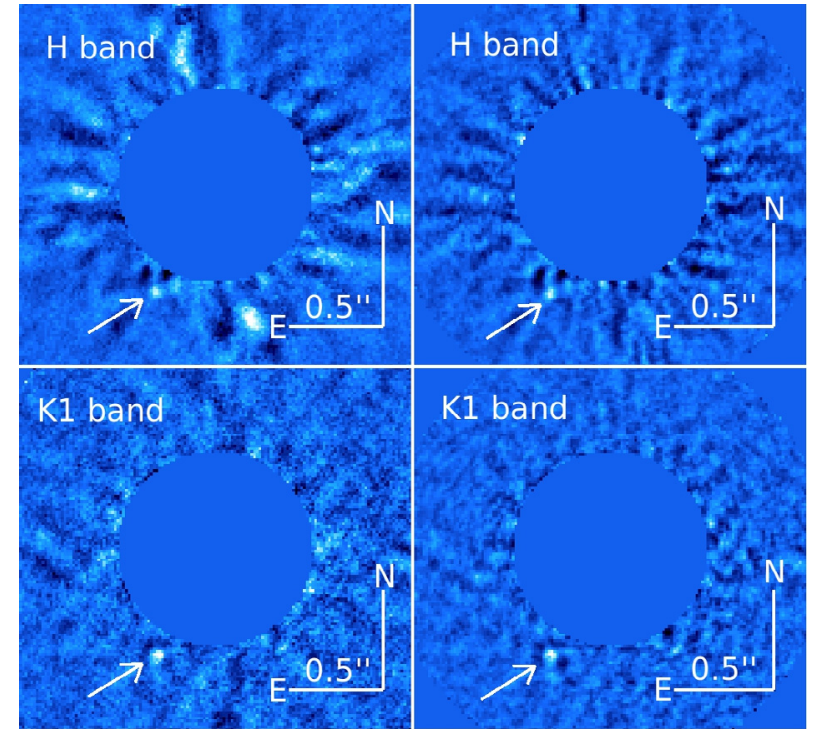

Fig. 1. Final images of the HD 95086 system at $H\left(\right.$ top ) and $K_{1}$ (bottom) bands from two of our pipelines. The planet (arrow) is detected in all images. The bright speckles are masked up to 500 mas from the central star.

cubes at $H$ and $K_{1}$ bands, respectively, consisting of 37 spectral channels each.

To further process the data, we registered each slice of the spectral cubes using the barycenter of the four satellite spots (attenuated replica of the central star PSF induced by a grid placed in a pupil plane, Marois et al. 2006b). Then, we minimized the speckle noise in each slice using independent pipelines each adopting various methods (Marois et al. 2006a; Lafrenière et al. 2007; Lagrange et al. 2010; Boccaletti et al. 2012; Chauvin et al. 2012; Soummer et al. 2012; Currie et al. 2013; Marois et al. 2014) used for ADI and spectral differential imaging (SSDI, Racine et al. 1999). Finally, all slices were mean-combined to yield an integrated broad-band image to maximize the signal-to-noise ratio $(\mathrm{S} / \mathrm{N})$ of any off-axis source. Binning images in wavelength and suppressing the speckles (ADI), or suppressing the speckles in each spectral channel (ADI/ADI+SSDI) and binning images give similar results, and all our pipelines recover HD 95086 b, which is the sole bright spot at the expected separation. Thus, we provide the first detections at $H$ and $K_{1}$ bands (Fig. 1) with an $\mathrm{S} / \mathrm{N}$ of $\sim 3-4$ and 5-6, respectively. The central bright speckles are masked up to 500 mas. These are the first detections of HD 95086 b with an instrument that is not $\mathrm{NaCo} / \mathrm{VLT}$. No spectrum can be extracted given the low $\mathrm{S} / \mathrm{N}$.

To estimate the planet flux and position, we needed unsaturated GPI PSFs. As GPI cannot acquire off-axis observations of the star, we calibrated photometry and astrometry using the satellite spots, which are expected to have same shape and brightness for a given filter. In the laboratory the spot-to-central-star flux ratios were $2.035 \times 10^{-4}(9.23 \mathrm{mag})$

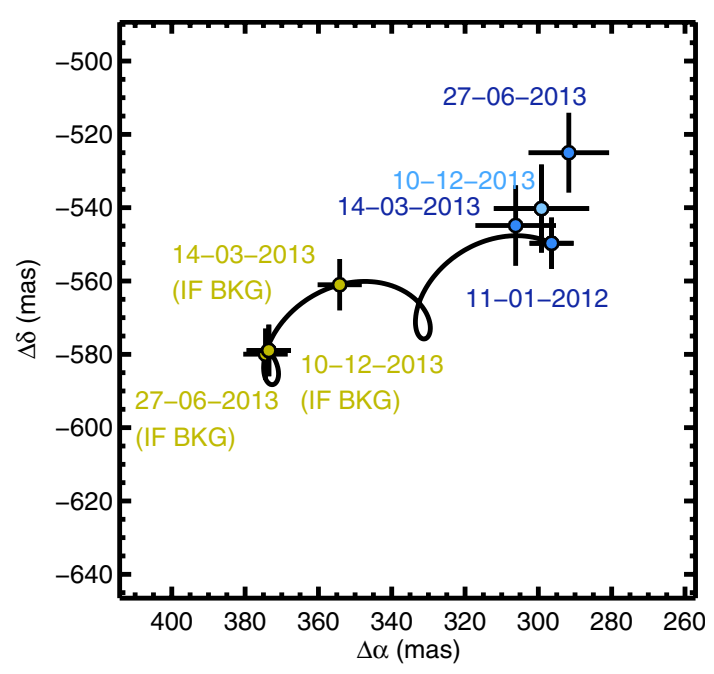

Fig. 2. HD $95086 \mathrm{~b}$ positions from its star in RA $(\Delta \alpha)$ and Dec $(\Delta \delta)$. GPI and $\mathrm{NaCo}$ measurements are marked in blue and expected positions of a background object in yellow.

and $2.695 \times 10^{-4}(8.92 \mathrm{mag})$ at $H$ and $K_{1}$ bands $^{1}$. To check these values, we compared $\mathrm{H}$ and $\mathrm{K}$ photometry of HD $8049 \mathrm{~B}$ (VLT/ NACO-SINFONI, Zurlo et al. 2013) with our measurements derived from public GPI HD 8049 data. Assuming that the object is not photometrically variable with time and considering the laboratory spot contrasts, GPI and VLT photometry are consistent within $\epsilon_{1}=0.2 \mathrm{mag}$, which we take as the error on the ratios. From these ratios, we assessed biases induced by our processing by injecting fake point-sources (i.e., unsaturated PSFs) into the data before applying speckle-suppression techniques (Lagrange et al. 2010; Marois et al. 2010b; Chauvin et al. 2012; Galicher et al. 2012). We obtained templates of the planet image. Adjusting the flux of the templates, we found the planet photometry and the fitting error $\epsilon_{2}$, which depends on the detection quality. $\epsilon_{2}$ is $0.8 \mathrm{mag}$ and $0.3 \mathrm{mag}$ at $H$ and $K_{1}$. Finally, we estimated the variation $\epsilon_{3}$ of stellar flux over the sequence with the variation of spot flux. $\epsilon_{3}$ is $0.2 \mathrm{mag}$ and $0.3 \mathrm{mag}$ over the $H$ and $K_{1}$ sequences including the variations between spots. The resulting photometric error is the quadratic error, which is dominated by the low $\mathrm{S} / \mathrm{N}$ at $H$ and is a mix of all errors at $K_{1}$.

For the astrometric error, we considered uncertainties in the centroiding accuracy of individual slices $(\leq 0.3$ pixel), the plate scale $(0.02$ pixel $)$, the planet template fit $(0.7$ pixel at $H, 0.5$ pixel at $\left.K_{1}\right)$, and the North position angle $(1 \mathrm{deg})$. The error is dominated by the low $\mathrm{S} / \mathrm{N}$ of the detections and the generic GPI calibrations. The current precision is good enough to assess the comoving status of the companion (Fig. 2). We tried to use the astrometric standard HD 8049 B in GPI data to better constrain the North orientation. We did not succeed because of the high orbital motion of HD $8049 \mathrm{~B}$ and because there is no contemporary observation from other instruments. 
R. Galicher et al.: HD 95086 GPI follow-up

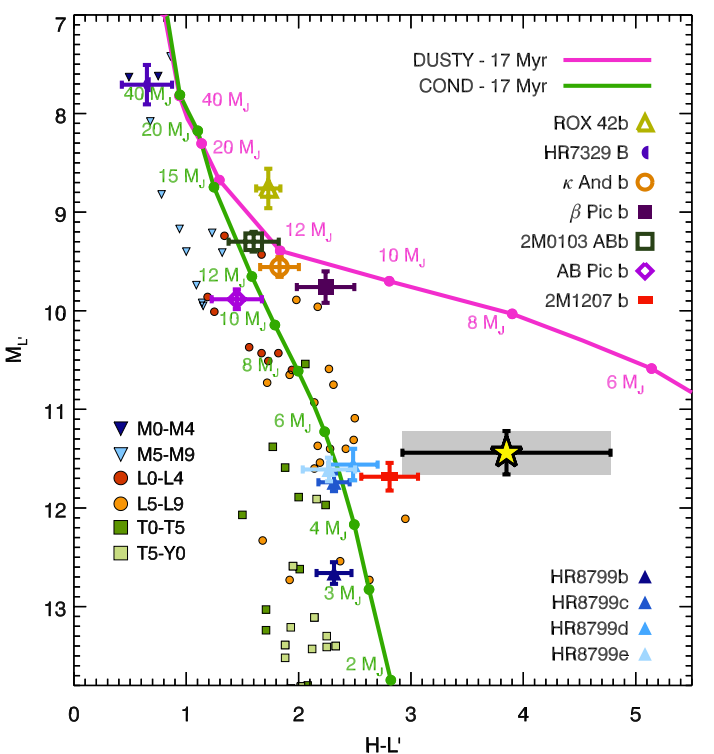

Fig. 3. Color-magnitude diagram using the new $H$-band photometry of HD 95086 b (yellow star) and data from Bonnefoy et al. (2013), Bonnefoy et al. (2014), and Currie et al. (2014).

Table 2. HD $95086 \mathrm{~b}$ photometry and astrometry at $H$ and $K_{1}$ (GPI data) and $L^{\prime}$ (Rameau et al. 2013a,b, and revision*).

\begin{tabular}{lcccc}
\hline \hline Date & Filter & Sep $(\mathrm{mas})$ & $\mathrm{PA}\left({ }^{\circ}\right)$ & $\Delta m$ \\
\hline $2013 / 12 / 11$ & $H$ & $633 \pm 17$ & $150.6 \pm 1.7$ & $13.1 \pm 0.9$ \\
$2013 / 12 / 10$ & $K_{1}$ & $623 \pm 15$ & $151.4 \pm 1.5$ & $12.1 \pm 0.5$ \\
$2013 / 03 / 24$ & $L^{\prime}$ & $626.1 \pm 12.8$ & $150.7 \pm 1.3$ & $9.71 \pm 0.56$ \\
$2012 / 01 / 11$ & $L^{\prime}$ & $623.9 \pm 7.4$ & $151.8 \pm 0.8$ & $9.79 \pm 0.40$ \\
$2012 / 01 / 11$ & $L^{\prime}$ & $623.9 \pm 7.4$ & $151.8 \pm 0.8$ & $9.48 \pm 0.19^{*}$ \\
\hline
\end{tabular}

Notes. ${ }^{(*)}$ See text for details.

Final measurements are presented in Table 2. We include revised $2012 \mathrm{NaCo} L^{\prime}$ photometry obtained by 1) better calibrating the planet signal (as in Currie et al. 2013) and 2) precisely deriving the $L^{\prime}$ neutral density (ND) filter throughput (used to flux-calibrate HD 95086) by comparing ND and unsaturated $\beta$ Pic data.

\section{Characterization}

Absolute magnitudes were derived from the contrast ratios (Table 2): $M_{H}=15.29 \pm 0.91 \mathrm{mag}, M_{K_{1}}=14.11 \pm 0.51 \mathrm{mag}$, and $M_{L^{\prime}}=11.44 \pm 0.22 \mathrm{mag}$ using the 2MASS and WISE W1 (Cutri et al. 2003, 2012) photometry of the star $^{2}$.

Combining the $H$ band GPI data with revised $\mathrm{NaCo} L^{\prime}$ data, we compared the $L^{\prime} / H-L^{\prime}$ color-magnitude diagram position of HD 95086b with that of young companions, field dwarfs (Leggett et al. 2010, 2013), and LYON evolutionary tracks (Chabrier et al. 2000; Baraffe et al. 2003) generated for the GPI/NaCo passbands ${ }^{3}$.

We converted the GPI measurements into $\mathrm{H}$ photometry by applying correction factors derived from published spectra, the filter transmissions, and a spectrum of Vega. HD 95086 b lies at

\footnotetext{
2 Correction factors from the GPI/NaCo and 2MASS/WISE photometry, derived from the spectrum of an A7III star in the Pickles et al. (1998) library, are negligible.

3 http://phoenix.ens-lyon. fr/simulator/index. faces
}

Table 3. Physical parameters predicted by hot-start evolutionary models for the observed absolute magnitudes.

\begin{tabular}{lcccc}
\hline \hline & \multicolumn{2}{c}{ SED } & \multicolumn{2}{c}{$L^{\prime}$} \\
Model & BT-SETTL & Lesia & Dusty & Cond \\
\hline$T_{\text {eff }}(\mathrm{K})$ & $1050_{-450}^{+450}$ & $1200_{-300}^{+300}$ & $916_{-44}^{+43}$ & $1108_{-65}^{+66}$ \\
$\log g($ dex $)$ & $4.0_{-0.5}^{+0.5}$ & $3.3_{-1.2}^{+1.2}$ & $3.8_{-0.1}^{+0.1}$ & $3.9_{-0.1}^{+0.1}$ \\
$M\left(M_{\text {Jup }}\right)$ & - & - & $4.5_{-1}^{+1}$ & $5.5_{-1.5}^{+1.5}$ \\
\hline
\end{tabular}

the $L-T$ transition in this diagram, similar to other young (8$30 \mathrm{Myr}$ ) planets like HR 8799 cde (Marois et al. 2008, 2010a) and 2M1207b (Chauvin et al. 2004). Its red $H-L^{\prime}$ color compared with the sequence of field dwarf objects (Leggett et al. 2010, 2013) suggests a high content of photospheric dust (Barman et al. 2011; Currie et al. 2011), owing to reduced surface gravity (e.g. Fig. 11 of Marley et al. 2012).

We built the $1.5-4.8 \mu \mathrm{m}$ spectral energy distribution (SED) of the planet following Bonnefoy et al. (2013) by combining the GPI photometry with the $L^{\prime}$ one. The normalized SED (at $L^{\prime}$ ) is best compatible with the young exoplanets HR 8799 bcde and $2 \mathrm{M} 1207 \mathrm{~b}$, but is redder. Its colors are also $\sim 1$ mag redder than those of the benchmark dusty L6.5-L7.5 field dwarf 2MASS J22443167+2043433 (Dahn et al. 2002; Stephens et al. 2009).

We also compared the SED of HD $95086 \mathrm{~b}$ with the predictions from grids of synthetic spectra for BT-SETTL (Allard et al. 2012) and LESIA atmospheric models (Baudino et al. 2014). Each synthetic SED was normalized to that of HD 95086 b at $L^{\prime}$. The BT-SETTL grid covers $400 \mathrm{~K} \leq T_{\text {eff }} \leq 3500 \mathrm{~K}$ with 50 to $100 \mathrm{~K}$ increments, $-0.5 \leq \log \mathrm{g} \leq 6.0 \mathrm{dex}$ with $0.5 \mathrm{dex}$ increments, and $M / H=0.0$ or +0.5 dex. The BT-SETTL models that reproduce the photometry of HD $95086 \mathrm{~b}$ have $600 \mathrm{~K} \leq T_{\text {eff }} \leq$ $1500 \mathrm{~K}$ and $3.5 \mathrm{dex} \leq \log g \leq 4.5 \mathrm{dex}$. The three LESIA grids assume $700 \mathrm{~K} \leq T_{\text {eff }} \leq 2100 \mathrm{~K}, 2.1 \leq \log \mathrm{g} \leq 4.5 \mathrm{dex}$, and solar abundances: one without clouds and two with clouds of $\mathrm{Fe}$ and $\mathrm{Mg}_{2} \mathrm{SiO}_{4}$ particles. For each LESIA model, we selected the planet radius that minimizes $\chi^{2}$ between the observed and calculated apparent magnitudes. We only kept models with a radius in a realistic range derived from evolution models ( 0.6 to 2 Jupiter radii, Mordasini et al. 2012). All LESIA models that reproduced the HD 950866 b photometry have $900 \mathrm{~K} \leq T_{\text {eff }} \leq 1500 \mathrm{~K}$ and 2.1 dex $\leq \log g \leq 4.5$ dex.

The planet mass cannot be derived from the atmosphere models, and evolutionary models are needed. Comparing the planet's $L^{\prime}$ luminosity with hot-start DUSTY and COND models for an age of $17 \pm 4 \mathrm{Myr}$, we find a planet mass of $M=5 \pm$ $2 M_{\text {Jup }}$ (Table 3). We did not use the $H$ and $K_{1}$ photometries because they are poorly reproduced by the models for an object at the $L-T$ transition (larger uncertainties than for $L^{\prime}$ ). The models predict $T_{\text {eff }}$ and $\log g$, in agreement with those derived from the SED fit.

Alternatively, we used the warm-start models (Spiegel \& Burrows 2012) to account for possible different initial conditions for the planet (parameterized by the initial entropy between 8 and $13 k_{\mathrm{B}}$ /baryon). The models assume solar metallicity and atmospheres enriched by a factor of 3 with/without dust clouds as boundary conditions. Synthetic SEDs are generated from predicted spectra of planets ${ }^{4}$. For the full range of initial entropies we considered, models assuming masses of 4-14 $M_{\mathrm{Jup}}$

\footnotetext{
4 http://www.astro.princeton. edu/ burrows/warmstart/ spectra.tar.gz
} 


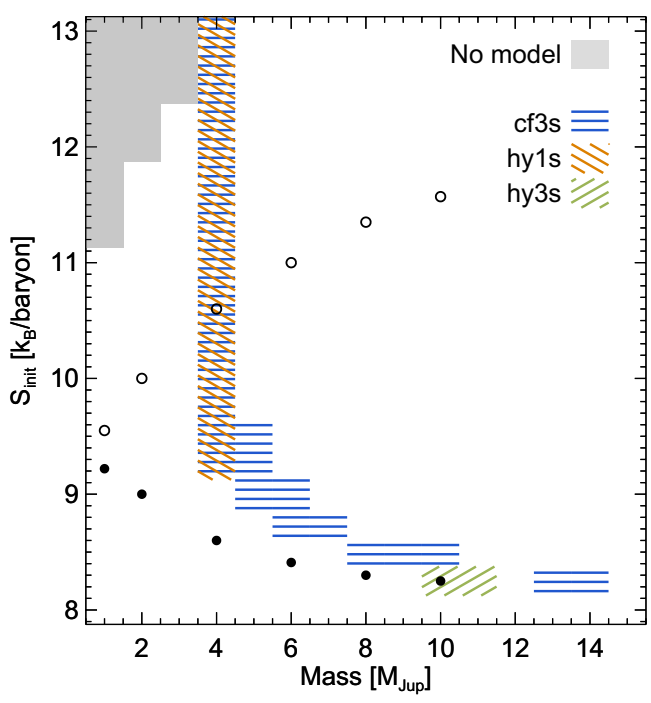

Fig. 4. Combination of initial entropies $\left(S_{\text {init }}\right)$ and masses (shaded areas) for which the planet 1.6-4.8 $\mu \mathrm{m}$ photometries are reproduced by the warm-start models of Spiegel \& Burrows (2012) within $1 \sigma$. Three boundary conditions are considered: with (hy) and without (cf) cloudy atmospheres, at solar (1s) and 3x solar (3s) metallicity. Initial entropies for the cold-start (filled circles) and hot-start (open circles) models of Marley et al. (2007) are overlaid.

match the SED of HD 95086b (Fig. 4). For much of this range $\left(S_{\text {init }}=9.5-13\right)$ a mass of $4 M_{\text {Jup }}$ is favored.

\section{Conclusions}

We reported the near-IR detections of HD 95086b from GPI public commissioning data. We confirmed that the companion is comoving with HD 95086 and derived the first estimates of its magnitudes with respect to its star: $H=13.1 \pm 0.9$ and $K_{1}=12.1 \pm 0.5$.

While the mid-IR luminosity of HD $95086 \mathrm{~b}$ is best consistent with an $L-T$ transition object, it has redder near-IR colors than other young, imaged planet-mass companions. This is consistent with a very dusty and low surface gravity atmosphere.

Comparison with atmosphere models provided $600 \mathrm{~K} \leq$ $T_{\text {eff }} \leq 1500 \mathrm{~K}$ and $2.1 \mathrm{dex} \leq \log g \leq 4.5 \mathrm{dex}$. Evolutionary models are consistent with a mass of $5 \pm 2 M_{\text {Jup }}$. However, the models are affected by systematic errors that are difficult to quantify because of the lack of young objects at the $L-T$ transition.

More higher-precision spectroscopic and photometric data for HD $95086 \mathrm{~b}$ are required to refine the planet properties.

Acknowledgements. We thank the consortium who built the GPI instrument and the data analysis team for developing reduction tools. We are grateful to Dave Spiegel and Adam Burrows for making the warm-start models publicly available. J.R., M.B., G.C., and A.M.L. acknowledge financial support from the French National Research Agency (ANR) through project grant ANR10BLANC0504-01. This research has benefitted from the SpeX Prism Spectral
Libraries, maintained by Adam Burgasser at http: //pono.ucsd.edu/ adam/ browndwarfs/spexprism. J.L.B.'s PhD is funded by the LabEx "Exploration Spatiale des Environnements Planétaires" (ESEP) \# 2011-LABX-030. T.C. is supported by a McLean Postdoctoral Fellowship.

\section{References}

Allard, F., Batten, A., Budding, E., et al. 2012, IAU Proc. 282, eds. M. T. Richards, \& I. Hubeny, 235

Baraffe, I., Chabrier, G., Barman, T., Allard, F., \& Hauschildt, P. 2003, A\&A, 402, 701

Barman, T., Macintosh, B., Konopacky, Q. M., \& Marois, C. 2011, ApJ, 735, L39

Baudino, J.-L., Brézard, B., Boccaletti, A., Bonnefoy, M., \& Lagrange, A.-M. 2014, IAU Proc., 299, 277

Boccaletti, A., Augereau, J.-C., Lagrange, A.-M., et al. 2012, A\&A, 544, A85

Bonnefoy, M., Boccaletti, A., Lagrange, A.-M., et al. 2013, A\&A, 555, A107

Bonnefoy, M., Chauvin, G., Lagrange, A.-M., et al. 2014, A\&A, 562, A127

Chabrier, G., Baraffe, I., Allard, F., \& Hauschildt, P. 2000, ApJ, 542, 464

Chauvin, G., Lagrange, A.-M., Dumas, C., et al. 2004, A\&A, 425, 29

Chauvin, G., Lagrange, A.-M., Beust, H., et al. 2012, A\&A, 542, A41

Chun, M., Toomey, D., Wahhaj, Z., et al. 2008, Proc. SPIE, 7015, 70151

Currie, T., Burrows, A., Itoh, Y., et al. 2011, ApJ, 729, 128

Currie, T., Burrows, A., Madhusudhan, N., et al. 2013, ApJ, 776, 15

Currie, T., Daemgen, S., Debes, J. H., et al. 2014, ApJ, 780, L30

Cutri, R., Skrutskie, M., Van Dyk, S., et al. 2003, VizieR On-line Data Catalog: II $/ 246$

Cutri, R., Skrutskie, M., Van Dyk, S., et al. 2012, VizieR On-line Data Catalog: II/311

Dahn, C., Harris, H., Vrba, F., et al. 2002, ApJ, 124, 1170

Galicher, R., \& Marois, C. 2012, Proc. of the AO4ELT conference, 25

Lafrenière, D., Marois, C., Doyon, R., Nadeau, D., \& Artigau, É. 2007, ApJ, 660,770

Lagrange, A.-M., Bonnefoy, M., Chauvin, G., et al. 2010, Science, 329, 57

Leggett, S., Burningham, B., Saumon, D., et al. 2010, ApJ, 710, 1627

Leggett, S., Morley, C., Marley, M., et al. 2013, ApJ, 763, 130

Lenzen, R., Hartung, M., Brandner, W., et al. 2003, Proc. SPIE, 4841, 944

Marley, M., Fortney, J., Hubickyj, O., Bodenheimer, P., \& Lissauer, J. 2007, ApJ, 655,541

Marley, M., Saumon, D., Cushing, M. et al. 2012, ApJ, 754, 135

Marois, C., Lafrenière, D., Doyon, R., Macintosh, B., \& Nadeau, D. 2006a, ApJ, 641, 556

Marois, C., Lafrenière, D., Macintosh, B., \& Doyon, R. 2006b, ApJ, 647, 612

Marois, C., Macintosh, B., Barman, T., et al. 2008, Science, 322, 1348

Marois, C., Macintosh, B., \& Véran, J.-P. 2010a, Proc. SPIE, 7736, 77361

Marois, C., Zuckerman, B., Konopacky, Q., et al. 2010b, Nature, 468, 1080

Marois, C., Correia, C., Véran, J.-P., \& Currie, T. 2014, IAU Symp., 299, 48

Macintosh, B., Graham, J., Ingraham, P., et al. 2014, PNAS, accepted [arXiv: 1403.7520]

Meshkat, T., Bailey, V., Rameau, J., et al. 2013, ApJ, 775, L40

Mordasini, C., Alibert, Y., Georgy, C., et al. 2012, A\&A, 547, A112

Pecaut, M.-J., Mamajek, E. E., \& Bubar, E. J. 2012, ApJ, 746, 154

Perrin, M., \& GPI Instrument and Science teams 2014, AAS Meeting Abstracts, 223, 348

Pickles 1998, PASP, 110, 863

Racine, R., Nadeau, D. \& Doyon, R. 1999, ESO Conf. Proc., 56, 377

Rameau, J., Chauvin, G., Lagrange, A.-M., et al. 2013a, ApJ, 772, L15

Rameau, J., Chauvin, G., Lagrange, A.-M., et al. 2013b, ApJ, 779, L26

Rousset, G., Lacombe, F., Puget, P., et al. 2003, Proc. SPIE, 4839, 140

Soummer, R., Pueyo, L., \& Larkin, J. 2012, ApJ, 755, L28

Spiegel, D., \& Burrows, A. 2012, ApJ, 745, 174

Stephens, D., Leggett, S., Cushing, M., et al. 2009, ApJ, 702, 154

van Leeuwen, F. 2007, A\&A, 474, 653

Zurlo, A., Vigan, A., Hagelberg, J., et al. 2013, A\&A, 554., A21 